\title{
Cellulose synthesis in coloured cotton
}

\author{
Sen-He Qian ${ }^{\mathrm{a}, \mathrm{b}}$, Liang Hong ${ }^{\mathrm{a}}$, Miao Xu $\mathrm{Xu}^{\mathrm{a}}$, Yong-Ping Cai ${ }^{\mathrm{a}}$, Yi Lin ${ }^{\mathrm{a}, *}$, Jun-Shan Gao \\ a College of Life Science, Anhui Agricultural University, Hefei 230036 China \\ b Dept. of Biochemistry, Anhui Polytechnic University, Wuhu 241000 China \\ ${ }^{*}$ Corresponding author, e-mail: linyi3000@163.com
}

Received 16 Apr 2014

Accepted 9 Jul 2015

\begin{abstract}
Using green, brown, and white cotton as materials, the contents of cellulose, carbohydrates, and flavonoid compounds as well as enzyme activities related to cellulose synthesis were investigated at various stages of fibre development to elucidate the characteristics of cellulose synthesis. The results showed that cellulose contents in all three cotton fibres showed 'S-shaped curve' changing patterns. According to a logistic growth model, the lower maximum accumulative rate of cellulose in the coloured cotton indicated less cellulose accumulation during fibre development. No significant differences in the carbohydrate content and carbohydrate transformation rates were observed between coloured and white cotton fibre. However, the flavonoid content in coloured cotton fibres was significantly higher than white cotton, and the flavonoid biosynthesis in coloured cotton fibres consumed a large amount of carbohydrates which should normally be used for cellulose synthesis. Low indoleacetic acid oxidase and peroxidase activities lowered cellulose accumulation in coloured cotton fibres. High activities of sucrose synthetase and $\beta$-1,3-glucanase in coloured cotton fibres may promote efficient utilization of carbohydrates. However, carbohydrate metabolism in the coloured cotton fibre should not only be responsible for cellulose biosynthesis but also for flavonoid biosynthesis.
\end{abstract}

KEYWORDS: fibre development, flavonoid, carbohydrate, enzyme activities, logistic growth model

\section{INTRODUCTION}

Coloured cotton comprise fibres containing natural pigment. The fibre colour results from pigment deposition in cells during fibre differentiation and development. The content and distribution of pigment directly determine the colour characteristics of the fibre ${ }^{1}$. Hua et $\mathrm{al}^{2}$ showed that flavonoid compounds are the main components for fibre pigment in coloured cotton. Because of the poor fibre quality of coloured cotton, only brown and green varieties are used in production ${ }^{3}$. Scientists have attempted to cross white cotton with coloured cotton to improve fibre quality of coloured cotton, but the results were unsatisfactory ${ }^{4}$. The inferior quality of coloured cotton fibre is mainly due to short fibre length, bad fibre strength, and micronaire, especially for low cellulose contents. White cotton exhibits higher cellulose content compared to coloured cotton ${ }^{5,6}$.

The biosynthesis of cellulose may be related to the carbohydrate concentration and the transformation rate of carbohydrates in addition to their physiological and biochemical characteristics during fibre development ${ }^{7,8}$. Sucrose synthase (SuSy) can catalyse the transformation of sucrose into fructose and uridine diphosphate glucose (UDPG), which can be transported to the cellulose synthase catalytic subunit to provide a substrate for cellulose synthe$\operatorname{sis}^{9,10}$. During the fibre secondary wall thickening stages, large amounts of non-cellulosic material, namely $\beta$-1,3-dextran, are produced in the inner cell wall of the fibre ${ }^{11}$. $\beta$-1,3-glucanase can hydrolyse the covalent bond of $\beta$-1,3-dextran and transport sugar molecules to the non-reduction end of cellulose, which can promote the synthesis of fibrilla ${ }^{12}$. In addition, indoleacetic acid oxidase (IAAO) and peroxidase (POD) are two important enzymes related to cellulose accumulation in cotton fibre. High IAAO and POD activities can promote the decomposition of indoleacetic acid (IAA) and increase the level of abscisic acid (ABA) to IAA in fibre cells enhancing the accumulation of cellulose ${ }^{13,14}$.

At present, the reason for the lower cellulose contents in coloured cotton is not well understood. In this study, green, brown, and white cotton cultivars were used to compare the differences in carbohydrate contents, the key enzyme activities related to cellulose synthesis, and flavonoid contents during fibre development. The practical application of this work was to elucidate the relationship between the physiological characteristics of cellulose accumulation and flavonoid synthesis in differently coloured cotton fibres. 


\section{MATERIALS AND METHODS}

\section{Cotton fibres}

Three upland cotton genotypes, namely Zongcaixuan No. 1 (brown cotton), Lvcaixuan No. 1 (green cotton) and Simian No. 3 (white cotton), were cultivated in the experimental farm of Anhui Agricultural University, Hefei, China. The cultivars were planted in the same homogeneous field with a randomized block design and three field replications. Plant-toplant and row-to-row distances of 50 and $100 \mathrm{~cm}$, respectively, were maintained. The conventional practices of cultivation were adopted during the entire growing period. The cotton blossoms on the upper branch of plants developing normally and with no pests were tagged. Tagged bolls were harvested at $5,10,15,20,25,30,35$, and 40 days post-anthesis (DPA). Harvested bolls were stored at $-70{ }^{\circ} \mathrm{C}$ prior to determinations.

\section{Determination of carbohydrate and cellulose contents}

Fibre samples (0.25 g) were placed in test tubes, and $5 \mathrm{ml}$ of distilled water was then added. The fibre was distilled in boiling water 4 times with the first time lasting for $30 \mathrm{~min}$ and the other times lasting for $15 \mathrm{~min}$. The fibre solvent was then filtered, and distilled water was added to a final volume of $25 \mathrm{ml}$. Carbohydrates were determined by the anthrone method ${ }^{15}$.

The residue from extracting soluble carbohydrates was dried in the oven until the weight of the fibre was stable. The residue was hydrolysed with $80 \%(\mathrm{v} / \mathrm{v}) \mathrm{H}_{2} \mathrm{SO}_{4}$ for $30 \mathrm{~min}$ in cold water, and it was then diluted with distilled water. After filtering, distilled water was added to a final volume of $100 \mathrm{ml}$. The hydrolysis sugar content was determined in accordance with the method of soluble carbohydrates $^{16}$.

\section{Determination of enzyme activities}

SuSy and $\beta$-1,3-glucanase activities were determined by a previously described ${ }^{17}$. Four times the volume of extraction buffer (100 mM Tris- $\mathrm{HCl}$, $10 \mathrm{mM} \mathrm{MgCl}_{2}, 2 \mathrm{mM}$ EDTA, 2\%(v/v) ethylene glycol, and $20 \mathrm{mM}$ mercaptoethanol) was added to $0.5 \mathrm{~g}$ of fibre, and the fibre was ground in an ice bath. The sample was then centrifuged at $10000 \mathrm{~g}$ for $10 \mathrm{~min}$ at $4^{\circ} \mathrm{C}$, and the generated sucrose was determined by the fructose and UDPG colorimetric method. For $\beta$-1,3-glucanase activity, 5 times the volume of $0.05 \mathrm{M}$ (pH 5.0) sodium acetate buffer was added to $0.5 \mathrm{~g}$ of fibre, and the fibre was ground in an ice bath. After centrifugation at $10000 \mathrm{~g}$ for $15 \mathrm{~min}$ at $4^{\circ} \mathrm{C}$, the sugar that was released from laminaria was determined by the DNS method.

For analysis of IAAO activity, 5 times the volume of $0.01 \mathrm{M}(\mathrm{pH}$ 6.1) phosphate buffer was added to $0.5 \mathrm{~g}$ of fibre, and the fibre was ground in an ice bath. The sample was then centrifuged at $13200 \mathrm{~g}$ for $10 \mathrm{~min}$ at $4^{\circ} \mathrm{C}$, and the resulting supernatant was used as the enzyme solution. Enzyme activity was determined by the $\mathrm{FeCl}_{3}-\mathrm{HClO}_{4}$ reagent method ${ }^{18}$.

The determination of POD activity was done according to the guaiacum colorimetric method ${ }^{19}$. Briefly, $5 \mathrm{ml}$ of $0.05 \mathrm{M}$ (pH 8.7) phosphate buffer and a small amount of quartz sand were added to $0.5 \mathrm{~g}$ of fibre, and the fibre was ground in an ice bath. The sample was then centrifuged at $13200 \mathrm{~g}$ for $15 \mathrm{~min}$ at $4{ }^{\circ} \mathrm{C}$, and the resulting supernatant was used as the POD enzyme solution. The reaction system contained $2.9 \mathrm{ml}$ of $0.05 \mathrm{M}$ phosphate buffer, $1 \mathrm{ml}$ of $2 \%(\mathrm{v} / \mathrm{v}) \mathrm{H}_{2} \mathrm{O}_{2}, 1 \mathrm{ml}$ of $0.05 \mathrm{M}$ guaiacum, and $0.1 \mathrm{ml}$ of enzyme. The enzyme solution was inactivated in boiling water for 5 min as a blank control. The absorbance was measured at $470 \mathrm{~nm}$ with a spectrophotometer, and the absorbance change of 0.01 per min was considered the peroxidase activity unit.

\section{Determination of flavonoid contents}

Methanol $(20 \mathrm{ml})$ was added to $0.5 \mathrm{~g}$ of fibre, and the fibre was extracted for $1.5 \mathrm{~h}$ by the reflux method in a $75^{\circ} \mathrm{C}$ water bath. The fibre solvent was filtered using Whatman paper, and methanol solution was added to a volume of $25 \mathrm{ml}$. The sample solvent $(1 \mathrm{ml})$ was placed in a flask, and $30 \%(\mathrm{v} / \mathrm{v})$ ethanol was added to a volume of $5 \mathrm{ml}$. Then, $0.3 \mathrm{ml}$ of $\mathrm{NaNO}_{2} 5 \%(\mathrm{w} / \mathrm{v})$ was added, and the mixture was mixed thoroughly and placed on a bench for $5 \mathrm{~min}$ before $0.3 \mathrm{ml}$ of $\mathrm{Al}\left(\mathrm{NO}_{3}\right)_{3} \quad 10 \%$ (w/v) was added. After $6 \mathrm{~min}, 2 \mathrm{ml}$ of $1 \mathrm{~N} \mathrm{NaOH}$ was added, and then $30 \%(\mathrm{v} / \mathrm{v})$ ethanol was added to a final volume of $10 \mathrm{ml}$. The absorbance was measured at $510 \mathrm{~nm}$ after the sample was placed on a bench for $10 \mathrm{~min}^{20}$.

\section{Data analysis}

The graphs were generated using ExCEL. The variance analysis (ANOVA) was performed with SAS 9.1. Logistic equations were analysed with SPSS 19.

The cellulose accumulation was described by the logistic growth model: $Y=Y_{\mathrm{a}} /\left(1+m \mathrm{e}^{k t}\right)$, where $t$ is the DPA (d), $Y$ is the cellulose content (\%), $Y_{\text {a }}$ is the maximum cellulose content (\%), and $m$ and $k$ are growth parameters. 


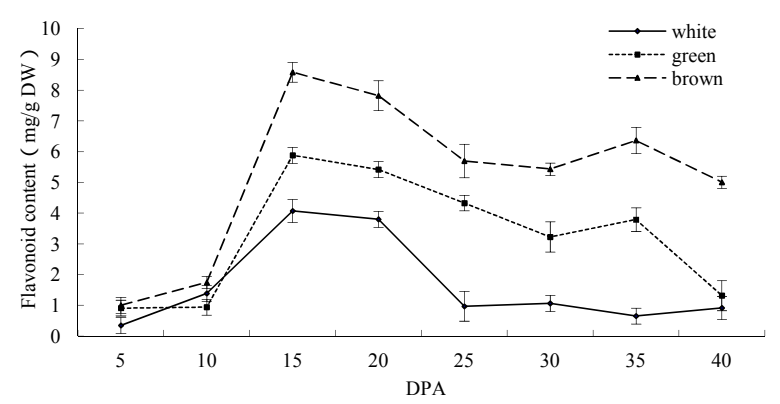

Fig. 1 Flavonoid contents in differently coloured fibres at various growth stages. DW, dry weight.

The parameters of $T_{1}$ (start time of cellulose rapid accumulation), $T_{2}$ (terminal time of cellulose rapid accumulation), and $V_{\max }$ (maximum accumulative rate of cellulose) were derived from the following equations:

$T_{1}=\frac{1}{k} \ln \frac{2+\sqrt{3}}{m}, T_{2}=\frac{1}{k} \ln \frac{2-\sqrt{3}}{m}, V_{\max }=\frac{-k Y_{\mathrm{a}}}{4}$.

\section{RESULTS}

\section{Changes in flavonoid contents}

Fig. 1 shows a peak of flavonoid content at 15 DPA in white cotton fibre, which then gradually decreased. However, the flavonoid content in the coloured cotton fibres had a peak at 15 DPA in addition to another smaller peak at 35 DPA. From 15-35 DPA, the flavonoid contents were significantly higher in coloured cotton fibres compared to white cotton ( $F=52.30-174.23 ; p \leqslant 0.0047)$, but at $40 \mathrm{DPA}$, only brown cotton had a higher flavonoid content than white cotton $(F=159.06 ; p \leqslant 0.0062)$.

\section{Changes in cellulose contents}

The characteristics of cellulose accumulation in cotton fibres of different genotypes are shown in Fig. 2. The cellulose contents in three kinds of cotton fibres presented an "S-shaped curve" changing patterns that could be described by the logistic growth model (Fig. 2, Table 1). The cellulose contents in the

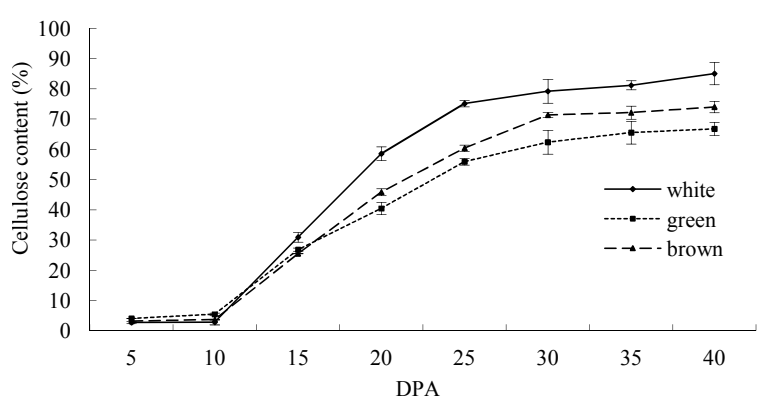

Fig. 2 Cellulose contents in differently coloured fibres at various growth stages.

coloured cotton were significantly lower compared to the white cotton at 40 DPA $(F=7.37 ; p=$ 0.0032). The maximum value of cellulose content in white, green, and brown cotton was 85, 67, and $74 \%$, respectively (Fig. 2). The biosynthesis of cellulose in white cotton sharply increased from 1025 DPA. However, the cellulose accumulation rate in brown cotton and green cotton were slower compared to white cotton, which led to lower cellulose contents in coloured cotton than in white cotton.

\section{Simulation equations and eigenvalues of cellulose contents}

The eigenvalues are presented in Table 1. The differences of cellulose accumulation characteristics among cotton fibres included the maximum accumulative rate of cellulose and the period length for rapid cellulose accumulation. Green cotton had the longest periods for rapid cellulose accumulation, and white cotton had the shortest periods. In contrast, the maximum accumulative rates of cellulose in coloured cotton were slower, but this rate was faster in white cotton fibre. In addition, coloured cotton entered the rapid cellulose accumulation phase one day earlier than the white cotton.

\section{Changes in carbohydrate contents}

The patterns of carbohydrate content were similar between coloured and white cotton fibres (Fig. 3).

Table 1 Simulation equations and eigenvalues of cellulose contents in differently coloured fibres.

\begin{tabular}{llcrrrrr}
\hline Cultivar & \multicolumn{1}{c}{ Model } & $R^{2}$ & $n$ & $T(\mathrm{~d})$ & $T_{1}(\mathrm{~d})$ & $T_{2}(\mathrm{~d})$ & $V_{\max }(\% / \mathrm{d})$ \\
\hline White cotton & $Y=81.956 /\left(1+244.412 \mathrm{e}^{-0.323 t}\right)$ & 0.994 & 8 & 8.2 & 12.9 & 21.1 & 6.615 \\
Green cotton & $Y=66.170 /\left(1+74.40 \mathrm{e}^{-0.243 t}\right)$ & 0.992 & 8 & 10.9 & 12.3 & 23.2 & 4.00 \\
Brown cotton & $Y=73.478 /\left(1+105.267 \mathrm{e}^{-0.257 t}\right)$ & 0.994 & 8 & 9.6 & 12.1 & 21.7 & 4.71 \\
\hline
\end{tabular}

$R^{2}=$ correlation coefficient, $n=$ number of cellulose contents used in equation, $T=$ period length for rapid cellulose accumulation; $T_{1}=$ start time of rapid cellulose accumulation; $T_{2}=$ terminal time of rapid cellulose accumulation; $V_{\max }=$ maximum accumulative rate of cellulose. 


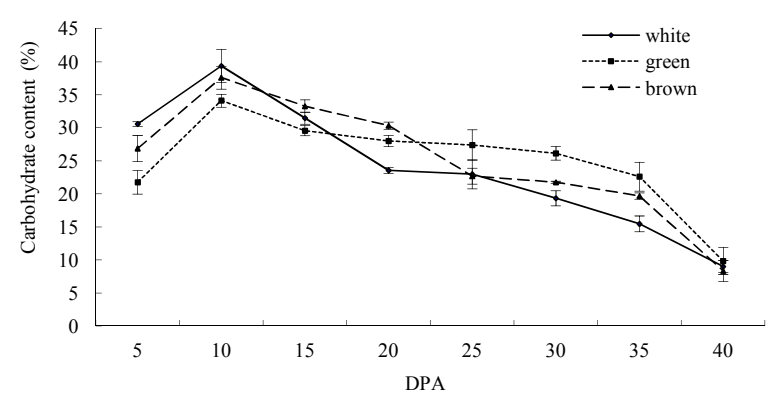

Fig. 3 Carbohydrate contents in differently coloured fibres at various growth stages. FW, fresh weight.

The carbohydrate contents presented as single-peak curves during fibre development. At 10 DPA, the white, brown, and green cotton fibres had the highest carbohydrate contents of 39,38 , and $34 \%$, respectively. However, no significant differences were observed between coloured and white cotton at this time point $(F=4.87 ; p=0.1142)$. From 10-40 DPA, the carbohydrate contents in all three fibres decreased continuously and gradually. The carbohydrate transformation rate of white cotton fibre $(78 \%)$ was higher than those of green and brown cotton (75 and $76 \%$, respectively), but there were also no significant differences among them $(F=2.39 ; p=0.2399)$.

\section{Changes in enzyme activities}

Fig. 4a shows the changes of fibre SuSy activity in the developmental stages for both white and coloured cotton cultivars. The decrease of SuSy activity of white cotton was found in the fibre developmental stage and declined sharply at 15-20 DPA. The SuSy enzyme activities of coloured cotton fibres sharply decreased at 5-15 DPA, increased gradually at 15-20 DPA, and then declined gradually again at 20-40 DPA. During fibre development, the SuSy activities in coloured cotton were significantly higher compared to white cotton ( $F=478.97-13.98 ; p \leqslant$ 0.0301 ), except at 15 DPA.

Similar patterns of fibre $\beta$-1,3-glucanase activities were observed in brown, green and white cotton (Fig. 4b). The fibre $\beta$-1,3-glucanase activities of coloured and white cotton were increased before 15 DPA and decreased after 15 DPA. During fibre development, the brown cotton had the highest $\beta-1,3$ glucanase activity, and the white cotton had the lowest $\beta$-1,3-glucanase activity. The $\beta$-1,3-glucanase activity was significantly higher in brown cotton fibre compared to white cotton during fibre development ( $F=26.59-530.37 ; p \leqslant 0.0356)$, except at 40 DPA.
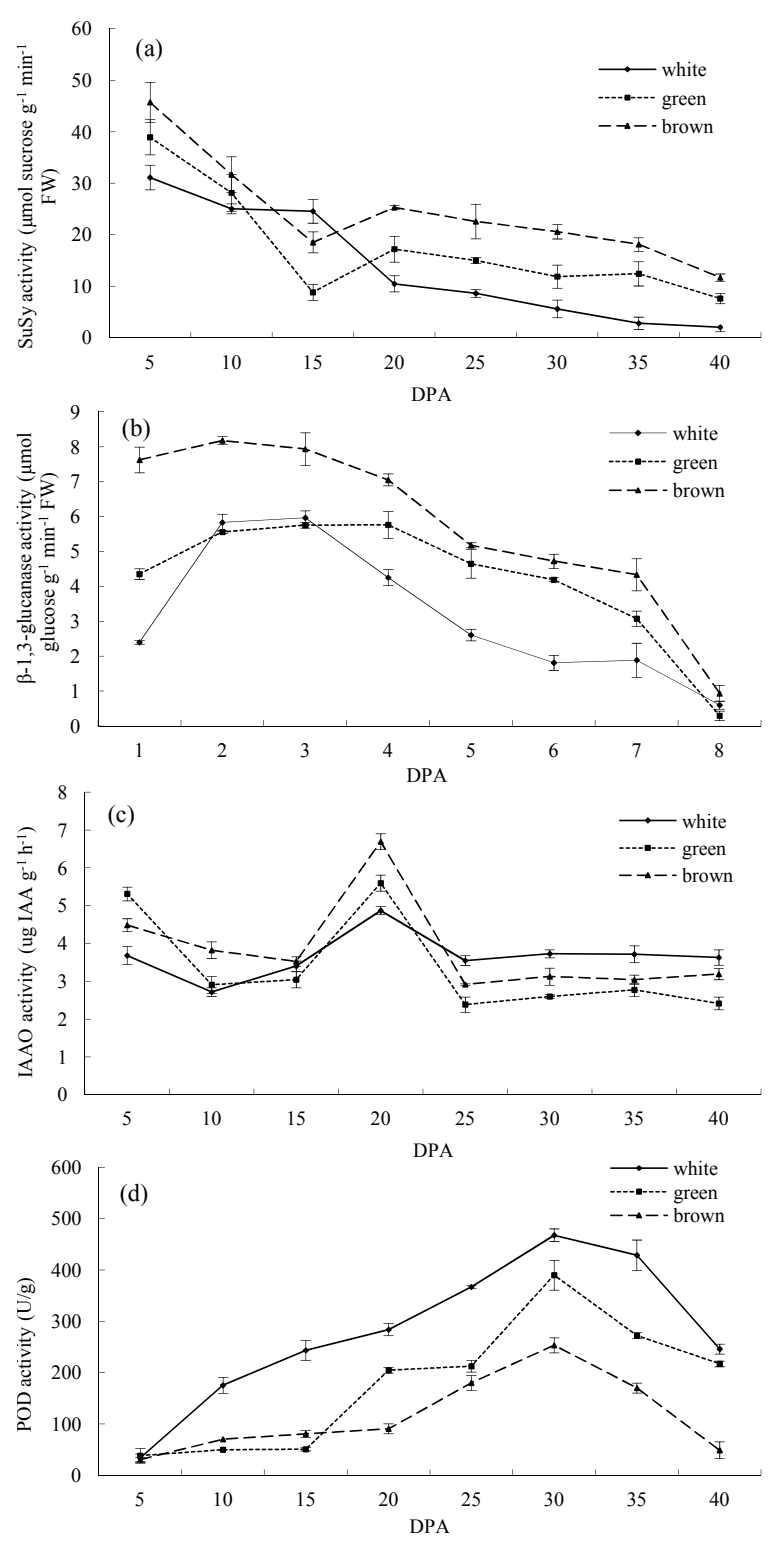

Fig. 4 Enzyme activity in differently coloured fibres at various growth stages: (a) SuSy, (b) $\beta$-1,3-glucanase, (c) IAAO, and (d) POD. FW, fresh weight.

For green cotton fibre, the $\beta$-1,3-glucanase activity was significantly higher than white cotton but only at $10,20,25$, and 30 DPA $(F=27.89-302.11 ; p \leqslant$ $0.034)$.

The IAAO activity changes during fibre development in each cotton cultivar are shown in Fig. 4c. The single-peak curves obtained at 20 DPA for fibre IAAO activities were similar between coloured and white cotton. At this time point, the IAAO activities in brown, green, and coloured cotton fibres were $6.69,5.60$, and $4.87 \mu \mathrm{g}$ IAA g ${ }^{-1} \mathrm{~h}^{-1}$, respectively. 
And the IAAO activities were significantly higher in coloured cotton fibres compared to white cotton fibre ( $F=21.27, p \leqslant 0.0169)$. The fibre IAAO activities in coloured cotton were higher before 20 DPA and significantly lower after 20 DPA $(F=11.35$ 49.07; $p \leqslant 0.0399$ ).

The general trends were similar among the white, brown, and green cotton fibres for changes in POD activities (Fig. 4d). The POD activities of all three fibres showed a single peak (enzyme activities were highest at the 30 DPA) followed by a decrease for all enzyme activities. The POD activity of white cotton fibre was significantly higher than that of coloured cotton ( $F=69.69-314.59, p \leqslant 0.0031$ ), except at 5 DPA.

\section{DISCUSSION}

Flavonoid compounds are important secondary metabolites that not only exist in young plant tissues but also in senescent organs ${ }^{21,22}$. Flavonoid synthesis is regulated by many factors, and the concentration of carbohydrates is one of the dominant factors ${ }^{20,23}$. Previous research has shown that flavonoid compounds are synthesized as the major pigments in colour cotton fibres ${ }^{2}$. Our results indicates that large amounts of flavonoid compounds were produced in coloured cotton fibres during fibre development, and their contents were significantly higher than that of white cotton (Fig. 1). We therefore conclude that a competition for carbohydrates between cellulose and flavonoid metabolic pathways exists during fibre development in coloured cotton.

Cellulose accumulation is responsible for the development of cotton fibre maturity and is important for the formation of fibre quality. Cellulose biosynthesis depends on the carbohydrate content and its transformation rate ${ }^{24}$. It is generally accepted that coloured cotton has lower cellulose contents when compared to white cotton ${ }^{20}$. Our results indicated that the white cotton fibre had the highest cellulose content and that green cotton had the lowest cellulose content. Moreover, the cellulose content in brown cotton was intermediate (Fig. 2, Table 1). A lower cellulose content might be an indication for lower carbohydrate content and carbohydrate transformation rate in coloured cotton fibre. Our findings indicate however that the differences of fibre carbohydrate contents and the carbohydrate transformation rates were not significant between coloured cotton and white cotton (Fig. 3), which may be due to the synthesis of flavonoid compounds consuming part of the carbohydrates that might be used for the synthesis of cellulose.

The logistic equation showed that there were some differences of the maximum accumulative rate of cellulose and the period length for rapid cellulose accumulation between coloured cotton and white cotton fibres (Table 1). The period length for rapid cellulose accumulation of coloured cotton was higher than that of white cotton (Table 1). In theory, a longer period for rapid cellulose accumulation should produce more cellulose in coloured cotton fibre ${ }^{25}$, but the maximum accumulative rate of cellulose in the coloured cotton fibres was much lower than that of white cotton fibre (Table 1). The lower accumulative rate of cellulose might be related to insufficient carbohydrate transformation during fibre development in the coloured cotton ${ }^{4}$.

In the cotton fibre development process, IAAO and POD have certain effects on the regulation of cellulose synthesis, including secondary wall thickening and elongation ${ }^{26}$. Low POD and IAAO activities restrain cell elongation during the fibre elongation stages of cotton ${ }^{27,28}$. During the secondary wall thickening stages, higher activities of POD and IAAO can accelerate the formation of fibre cell walls and promote steady cellulose accumulation ${ }^{14}$. The results of this study showed that the IAAO activity of colour cotton fibres was higher than that of white cotton from 5-25 DPA (Fig. 4c), which may inhibit fibre elongation. However, lower IAAO and POD activities during secondary cell wall synthesis stages in coloured cotton fibres had negative effects on cellulose accumulation (Fig. 4c,d).

SuSy and $\beta$-1,3-glucanase are two important enzymes involved in cellulose synthesis ${ }^{29,30}$. For fibre cells, SuSy can cleave sucrose into UDPG and fructose, and UDPG is then the main substrate for cellulose synthesis ${ }^{9}$. $\beta$-1,3-glucanase widely exists in higher plants, and $\beta$-1,3-glucanase plays an important role in plant defence responses ${ }^{31}$. However, in cotton fibre cells, $\beta-1,3$-glucanase is related to secondary wall thickening rather than a stress response. $\beta$-1,3-glucanase can digest $\beta$-1,3-dextran glycosidic bonds, hydrolyse callose, and provide UDPG for the synthesis of cellulose ${ }^{32}$. The onset of secondary wall thickening occurs at approximately 17 DPA. Thus 20-40 DPA are involved in the key stages for cellulose formation. In our experiments, the coloured cotton fibres had higher SuSy and $\beta$-1,3-glucanase activities than those of white cotton during later stages of fibre development (20-40 DPA) (Fig. 4c,d). Generally, higher activities of SuSy and $\beta$-1,3-glucanase are consistently correlated with high rates cellulose deposition in coloured cotton 
fibres ${ }^{28}$. Carbohydrate metabolism is however a complex process in coloured cotton fibre, and carbohydrates are not only used to synthesize cellulose but also to form flavonoid compounds ${ }^{33}$.

\section{Conclusions}

In summary, the final cellulose contents in the coloured cotton were significantly lower compared to that of the white cotton. Cellulose accumulation can be influenced by carbohydrate concentration, enzyme activities, and flavonoid biosynthesis in cotton fibre. The biosynthesis of flavonoid compounds may consume a part of the carbohydrates that might be used for cellulose synthesis. Lower IAAO and POD activities resulted in lower cellulose contents in coloured cotton fibres. However, higher SuSy and $\beta$-1,3-glucanase activities in coloured cotton fibres did not result in higher cellulose contents, which may be related to the competition for carbohydrates between cellulose biosynthesis and pigmentation.

Acknowledgements: The present investigation was financially supported by the grant from Anhui Cotton Industry Innovation System, The Biology Key Subject Construction of Anhui, and Educational Commission of Anhui Province (No. KJ2011A103; KJ2014A078).

\section{REFERENCES}

1. Richards AF, Rowe T, Stankovič Elesini U (2000) Structure of naturally coloured cottons. Int $J$ Polym Mater 47, 549-57.

2. Hua S, Wang X, Yuan S, Shao M, Zhao X, Zhu S, Jiang L (2007) Characterization of pigmentation and cellulose synthesis in colored cotton fibers. Crop Sci 47, 1540-6.

3. Murthy MSS (2001) Never say dye: The story of coloured cotton. Resonance 6, 29-35.

4. Yuan S, Hua S, Malik W, Bibi N, Wang X (2012) Physiological and biochemical dissection of fiber development in colored cotton. Euphytica 187, 215-26.

5. Delmer DP, Amor Y (1995) Cellulose biosynthesis. Plant Cell 7, 987-1000.

6. Martin LK, Haigler CH (2004) Cool temperature hinders flux from glucose to sucrose during cellulose synthesis in secondary wall stage cotton fibers. Cellulose 11, 339-49.

7. Zhang M, Song X, Sun X, Wang Z, Li Z, Ji H, Xu X, Li $J$ (2012) The relationship between cellulose content and the contents of sugars and minerals during fiber development in colored cotton cultivars. Cellulose 19, 2003-14.

8. Abidi N, Hequet E, Cabrales L (2010) Changes in sugar composition and cellulose content during the secondary cell wall biogenesis in cotton fibers. Cellulose 17, 153-60.
9. Salnikov VV, Grimson MJ, Seagull RW, Haigler $\mathrm{CH}$ (2003) Localization of sucrose synthase and callose in freeze-substituted secondary-wall-stage cotton fibers. Protoplasma 221, 175-84.

10. Delmer DP, Haigler CH (2002) The regulation of metabolic flux to cellulose, a major sink for carbon in plants. Metab Eng 4, 22-8.

11. Meier H, Buchs L, Buchala AJ, Homewood T (1981) $(1 \rightarrow 3)-\beta$-D-Glucan (callose) is a probable intermediate in biosynthesis of cellulose of cotton fibers. Nature 289, 821-2.

12. Saxena IM, Brown RM Jr (2000) Cellulose synthases and related enzymes. Curr Opin Plant Biol 3, 523-31.

13. Yang YM, Xu CN, Wang BM, Jia JZ (2001) Effects of plant growth regulators on secondary wall thickening of cotton fibres. Plant Growth Regul 35, 233-7.

14. Zhang WJ, Hu HB, Wang YH, Chen BL, Shu HM, Zhou ZG (2007) Fiber strength and enzyme activities of different cotton genotypes during fiber development. Sci Agric Sin 40, 2177-84, [in Chinese].

15. Li HS (2000) Experiment Principles and Techniques for Plant Physiology and Biochemistry, 1st edn, Higher Education Press, Beijing, [in Chinese].

16. Viles FJ Jr, Silverman L (1949) Determination of starch and cellulose with anthrone. Anal Chem 21, 950-3.

17. Tang ZC (1999) Modern Laboratory Manual of Plant Physiology, 1st edn, Science Press, Beijing, [in Chinese].

18. Jasdanwala RT, Sing YD, Chinoy JJ (1977) Auxin metabolism in developing cotton hairs. J Exp Bot 28, 1111-6.

19. Mäder M, Meyer Y, Bopp M (1975) Lokalisation der Peroxidase-Isoenzyme in Protoplasten und Zellwänden von Nicotiana tabacum L. Planta 122, 259-68.

20. Dutt Y, Wang XD, Zhu YG, Li YY (2004) Breeding for high yield and fibre quality in coloured cotton. Plant Breed 123, 145-51.

21. Ralston L, Subramanian S, Matsuno M, Yu O (2005) Partial reconstruction of flavonoid and isoflavonoid biosynthesis in yeast using soybean type I and type II chalcone isomerases. Plant Physiol 137, 1375-88.

22. Nishihara M, Nakatsuka T, Yamamura S (2005) Flavonoid components and flower color change in transgenic tobacco plants by suppression of chalcone isomerase gene. FEBS Lett 579, 6074-8.

23. Solfanelli C, Poggi A, Loreti E, Alpi A, Perata P (2006) Sucrose-specific induction of the anthocyanin biosynthetic pathway in Arabidopsis. Plant Physiol 140, 637-46.

24. Pettigrew WT (2001) Environmental effects on cotton fiber carbohydrate concentration and quality. Crop Sci 41, 1108-13.

25. Zhang W, Shu H, Hu H, Chen B, Wang Y, Zhou Z (2009) Genotypic differences in some physiological characteristics during cotton fiber thickening and its influence on fiber strength. Acta Physiol Plant 31, 
927-35.

26. Rao NR, Naithani SC, Jasdanwala RT, Singh YD (1982) Changes in indoleacetic acid oxidase and peroxidase activities during cotton fibre development. $Z$ Pflanzenphysiol 106, 157-65.

27. Delannoy E, Marmey P, Jalloul A, Etienne H, Nicole M (2006) Molecular analysis of class III peroxidases from cotton. $J$ Cotton Sci 10, 53-60.

28. Zhang W, Hu H, Chen B, Shu H, Wang Y, Zhou Z (2007) Genotypic differences in some physiological characteristics during cotton fiber thickening and its relationship with fiber strength. Acta Agron Sin 33, 531-8, [in Chinese].

29. Brown RM Jr, Saxena IM, Kudlicka K (1996) Cellulose biosynthesis in higher plants. Trends Plant Sci 1, 149-56.

30. Ruan YL, Llewellyn DJ, Furbank RT (2003) Suppression of sucrose synthase gene expression represses cotton fiber cell initiation, elongation, and seed development. Plant Cell 15, 952-64.

31. Saikia R, Singh BP, Kumar R, Arora DK (2005) Detection of pathogenesis-related proteins-chitinase and $\beta$-1,3-glucanase in induced chickpea. Curr Sci 89, 659-63.

32. Shimizu Y, Aotsuka S, Hasegawa O, Kawada T, Sakuno T, Sakai F, Hayashi T (1997) Changes in levels of mRNAs for cell wall-related enzymes in growing cotton fiber cells. Plant Cell Physiol 38, 375-8.

33. Winter H, Huber SC (2000) Regulation of sucrose metabolism in higher plants: localization and regulation of activity of key enzymes. Crit Rev Plant Sci $19,31-67$. 